\title{
Determination of thermodynamic and structural quantities of polymers by scattering techniques
}

https://doi.org/10.1515/pac-2017-1101

\begin{abstract}
Scattering techniques (i.e. light scattering, X-ray scattering, or neutron scattering) are very powerful tools to gain insights into structural and thermodynamic properties of matter which often cannot be obtained by other methods. While classical thermodynamics is independent of length scale or applies for indefinitely long length scale, scattering can disclose thermodynamic properties like the free energy or free enthalpy as functions of length scale. Scattering is caused by density or composition fluctuations, which are functions of the length scale in one- or multicomponent systems. Therefore scattering techniques can give informations about the size, shape and molecular weight of scattering objects, their thermodynamic interactions with a surrounding matrix and their dynamics if correlations of the fluctuations as function of time are investigated (i.e. dynamic light scattering). As scattering techniques are less intuitive in comparison to complementary techniques, i.e. microscopic techniques, the aim of this article is to highlight some relevant relationships with a focus on polymer systems. This may encourage polymer scientists to consider the use of scattering techniques to learn more about the thermodynamics of their systems and/or to gain informations about their structural properties.
\end{abstract}

Keywords: POLYCHAR-25; polymers; scattering techniques; structure; thermodynamic properties.

\section{Introduction}

Measurements of thermodynamic properties like an osmotic pressure or the change of a boiling point of a solvent in a dilute solution can give access to the molecular weight of a dissolved (macro)molecule and its interaction with the solvent. Scattering experiments can deliver in addition structural quantities such as the size and shape of dissolved macromolecules or colloids [1-9]. In this paper we will introduce the fundamental concepts of static as well as dynamic scattering experiments, which are in our opinion most relevant for characterization of polymers in solution and amorphous polymer systems. Therefore we discuss systems in which the basic structural units are on a length scale ranging from a repeating unit of a polymer up to the size of a macromolecule. We do not consider crystallographic structural investigations on the length scale of atoms.

An absolute homogeneous system does not scatter, because dissolved molecules or dispersed particles are indistinguishable from their surrounding (matrix). The prerequisite for scattering is a contrast between different components or the particles of interest and the matrix. The contrast is always based on a difference (fluctuation) between the corresponding scattering properties of the particle and the matrix. The type of

Article note: A collection of invited papers based on presentations at the $25^{\text {th }}$ POLYCHAR 2017 World Forum on Advanced Materials Kuala Lumpur, Malaysia, October 9-13, 2017.

\footnotetext{
*Corresponding author: Volker Abetz, University of Hamburg, Institute of Physical Chemistry, Grindelallee 117, 20146 Hamburg, Germany; and Helmholtz-Zentrum Geesthacht, Institute of Polymer Research, Max-Planck-Straße 1, 21502 Geesthacht, Germany, e-mail: volker.abetz@hzg.de. http://orcid.org/0000-0002-4840-6611

Birgit Fischer: University of Hamburg, Institute of Physical Chemistry, Grindelallee 117, 20146 Hamburg, Germany
} 
contrast depends on the probe (photons of light, X-ray photons, neutrons, electrons, $\alpha$-particles ...). While photons of visible light are scattered by fluctuations of the polarizability, which is related to fluctuations of the refractive index, X-ray photons are scattered by fluctuations of the electron density. Neutrons are scattered by the nuclei of atoms. The respective type of contrast is expressed in terms of a contrast factor which is given for these three types of probes in Table 1.

The fluctuations of these quantities can be related to the fluctuation of the density $\delta \rho$ or the concentration $\delta c$. By "fluctuation" a local deviation form a spatial average value is meant, i.e. the local concentration at space point $r, c(r)$ deviates by the fluctuation $\delta c(r)$ from the spatial average value $c$. Thus $\delta c(r):=c(r)-c$. Density and concentration fluctuations are related to fluctuations of the free energy $d F$ and the free enthalpy $d G$, respectively.

In scattering elastic and inelastic processes can occur. While in an elastic scattering event just the momentum of the probe is changed by changing its direction (i.e. Rayleigh scattering [10] or Mie scattering [11] in the case of light, or Rutherford scattering in the case of charged particles like $\alpha$-particles [12]), in an inelastic scattering event (i.e. Raman scattering [13, 14] or Compton scattering [15]) also energy is transferred between scatterers and probe resulting in a change of the wavelength of the scattered probe. In Raman spectroscopy energy of light is transferred to vibrating/rotating molecules (resulting in a so-called Stokes shift of the scattered light to longer wavelength) or internal energy is transferred from a vibrating/rotating molecule to the light (leading to a so-called Anti-Stokes shift of the scattered wavelength) [13]. While vibrational and rotational transitions lead to spectroscopically measurable shifts of the wavelength, the wavelength shifts caused by the Doppler effect of scattered light on diffusing particles is very small and the dynamic quantities are obtained from the time dependence of the fluctuating scattered intensity. This is why dynamic light scattering is considered to be quasi elastic scattering, as the energy transfer between light and scatterer is extremely low. Rayleigh scattering is observed for scatterers much smaller than the wavelength of the light (point-like scatterers like gases).

In case of dilute polymers in solution also Rayleigh scattering can be observed, as the macromolecules are typically not larger than ca. 1/20 of the wavelength of light. The elastic scattering of isolated spherically shaped colloidal nanoparticles with sizes in the range of the wavelength can be described by the Mie theory, which also explains the size dependence of the color [11].

However, a complete list of scattering methods would go beyond of the scope of this manuscript. For an understanding of the detailed mathematical concepts of wide angle X-ray diffraction, small angle X-ray scattering, and small angle light scattering we can refer to references $[1,5,16]$.

In static scattering experiments, the scattered intensity arises from local inhomogeneities (local fluctuations) in the scattering contrast and is monitored as a function of scattering angle. The particle shape, the molar mass $M_{W}$, the radius of gyration $R_{g}$ and the particle interaction can be determined. In dynamic scattering experiments the temporal fluctuations lead to a fluctuating scattered intensity at a fixed scattering angle

Table 1: Scattering contrast factor and typical wavelength for light, neutron and X-ray experiments.

\begin{tabular}{llll}
\hline & Light & Neutron & X-rays \\
\hline Contrast factor K & $\frac{4 \pi^{2} n_{s}^{2}}{\lambda_{0}^{4}}\left(\frac{d n}{d c_{m}}\right)^{2}$ & $\left(\sum_{i} b_{i}-\sum_{j} b_{j}\right)^{2} \frac{N_{L}}{M_{w}^{2}}$ & $r_{e}^{2}\left(\rho_{e, i}-\rho_{e, j}\right)^{2} v_{m}^{2} \frac{N_{L}}{M_{w}^{2}}$ \\
Wave length & $400-700 \mathrm{~nm}$ & $0.1-1 \mathrm{~nm}$ & $0.1-1 \mathrm{~nm}$ \\
Scattering vector & $3 \times 10^{-3}-3 \times 10^{-3} \mathrm{~nm}^{-1, \mathrm{a}}$ & $5 \times 10^{-2}-5 \mathrm{~nm}^{-1, \mathrm{~b}}$ & $5 \times 10^{-2}-5 \mathrm{~nm}^{-1, \mathrm{~b}}$ \\
\hline
\end{tabular}

Here $\lambda_{0}$ is the wavelength in vacuum, $n_{s}$ is the refractive index of the matrix (solvent), $\frac{d n}{d c_{m}}$ is the refractive index increment, $\Sigma b_{i}$ and $\Sigma b_{j}$ are the scattering lengths of the particle $i$ and the solvent $j, M_{w}$ is the weight averaged molecular weight, $r_{e}$ is the classical electron radius, $\rho_{e, i}$ and $\rho_{e, j}$ are the electron density of the particle $i$ and the solvent $j$ and $v_{m}$ is the volume. ${ }^{a}$ Depending on the solvent, for light scattering mostly a wavelength of about $633 \mathrm{~nm}$ is used. ${ }^{\mathrm{b} D e p e n d i n g}$ on the detector size, the detector distance and the used wavelength. 
which is monitored. The diffusion coefficient can be determined from such data and in the case of dynamic light scattering of dilute polymer solutions the hydrodynamic size of the polymers can be calculated.

The basic scheme of a scattering experiment is shown in Fig. 1. The incident beam passes through the sample and gets on the one hand absorbed and on the other hand scattered by the scattering objects of the sample. In a typical scattering experiment the scattering vector $\vec{q}=\overrightarrow{k_{f}}-\overrightarrow{k_{i}}$ is used, which is the difference between the wave vectors of the scattered and incident plane waves, $\overrightarrow{k_{f}}$ and $\overrightarrow{k_{i}}$. The intensity of the scattered beam $\overrightarrow{k_{f}}$ is observed at different scattering angles at a defined distance $A$ by a detector (D). The scattering angle $2 \theta$ is the angle between transmitted and scattered beams and can usually be tuned in an experiment. While a time-averaged constant scattered intensity is monitored in static scattering at each scattering angle, a time-resolved fluctuating intensity is monitored at each scattering angle in a dynamic scattering experiment.

In elastic scattering the wave vectors have the same length $\left(\left|\overrightarrow{k_{f}}\right|=\left|\overrightarrow{k_{i}}\right|\right)$ and in quasi elastic scattering the wave vectors have almost the same length $\left(\left|\overrightarrow{k_{f}}\right| \approx\left|\overrightarrow{k_{i}}\right|\right)$. Therefore in both cases the scattering vector $q$ is given by

$$
q=|\vec{q}|=\frac{4 \pi n_{0}}{\lambda} \sin (\theta)
$$

Here $n_{0}$ is the refractive index of the scattering medium, $\lambda$ the wavelength in vacuum and $2 \theta$ is the scattering angle, enclosed by $\vec{k}_{f}$ and $\vec{k}_{i}$ (Fig. 1).

Here we will discuss the structure factor in dilute polymer solutions and in polymer bulk systems, as it can be determined from static scattering of light, X-rays, and neutrons. This will be followed by a short discussion of dynamic light scattering of dilute polymer solutions.

\section{Static scattering of dilute solutions}

Polymer solutions and colloidal solutions can be well characterized by light scattering, small angle X-ray scattering (SAXS), and small angle neutron scattering (SANS) experiments.

In order to discuss the scattering results the scattering function $S(q)$, also called structure factor, structure function, or interference function is introduced, which can be calculated by dividing the measured scattered intensity $I(q)$ by the product of the scattering intensity of one repeating unit of the polymer [in the following called "monomer"] (from a chemical point of view this is incorrect, but here we follow the term mostly used by the physicists in this context) $I_{m}(q)$ and the total number of monomers in the sample $\mathcal{N}_{m}[1]$

$$
S(q) \propto \frac{I(q)}{I_{m}(q) \mathcal{N}_{m}} .
$$

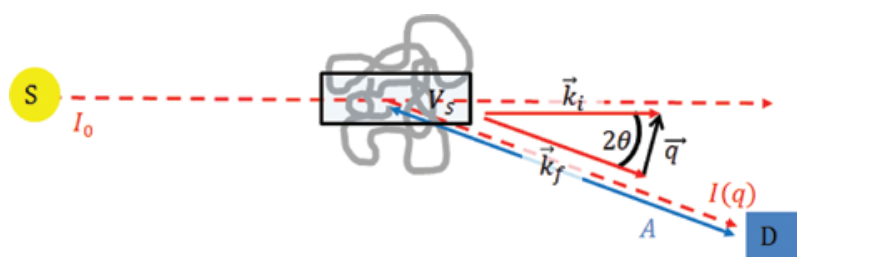

Fig. 1: Scheme of a typical scattering experiment. From a source $(S)$ an incident monochromatic beam characterized by the wave vector $\vec{k}_{i}$ with intensity $I_{0}$ and wavelength $\lambda$ passes through a sample through a volume $V_{S}$. The intensity of the scattered beam with a wave vector $\vec{k}_{f}$ is observed at different scattering angles at a defined distance $A$ by a detector (D). The scattering angle $2 \theta$ is the angle between transmitted and scattered beam. The scattering vector $\vec{q}$ is defined as $\vec{q}=\overrightarrow{k_{f}}-\vec{k}_{i}$. The incident waves $\vec{k}_{i}$ are in phase. The phase relation (interference) of the scattered beam depends on the distance between the scatterers and the angle

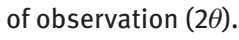


Typically the differential cross section per unit volume of the sample $\Sigma(q)$

$$
\sum(q)=\frac{1}{V} \frac{d \sigma}{d \Omega}=\frac{1}{V} \frac{I(q) A^{2}}{I_{0}}
$$

is measured. $(d \sigma / d \Omega)_{m}$ is the differential scattering cross section per monomer. In case of light scattering experiments it is typically called the Rayleigh ratio $\Sigma(q) \equiv R(q)$. The Rayleigh ratio is related to the interference function $S(q)$ by

$$
\sum(q)=\left\langle C_{m}\right\rangle\left(\frac{d \sigma}{d \Omega}\right)_{m} S(q)
$$

where $\left\langle c_{m}\right\rangle=\mathcal{N}_{m} / V$ is the mean number density of monomers.

The scattering of polymer solutions deviates from an ideal Rayleigh scattering due to two contributions. The first contribution results from the size of the scattering particle (individual polymer chain). Due to its size the polymer needs to be treated as an ensemble of many scattering centers with superposition of their scattering intensities (intraparticle interference). In highly diluted solutions the different polymers scatter independently from each other and the measured scattering intensity is the sum of the individual polymers in the irradiated volume (scattering volume). Therefore the phase relation (interference) of the scattered beam depends on the distance between the scatterers within the polymer and the angle of observation (Fig. 1). The second contribution results from interparticle interferences between different scattering particles if the concentration is not very low and interactions (correlations) between different scattering particles cannot be neglected. The structure factor introduced in eq. 5 combines both contributions from intra- and interparticle interferences. To distinguish these contributions, one can introduce the particle form factor $P(q)$ for the intraparticle interferences and an interparticle structure factor $S_{I P}(q)$ which describes the interparticle interferences [17].

For isotropic particles the relation for the measured intensity can then be factorized and simplified to [17]

$$
S(q)=N_{P} P(q) S_{I P}(q)
$$

If all particles scatter incoherently - like in a gas - the interparticle structure factor $S_{I P}(q)$ equals unity and the scattered intensity is proportional to the scattering of a single particle.

As prominent examples, the particle form factors $P(q)$ of a sphere, a random coil, and a rod are given in Table 2. In Fig. 2 these form factors are plotted as a function of a scaled scattering vector. As a scaling factor a characteristic length of the particles is chosen, like the radius of gyration $R_{g}$ in the case of spheres and coils, and the length $L$ in case of the rods. For simplicity, in the following discussion we only consider $R_{g}$ rather than $L$.

As shown in Fig. 2b, the inverse particle form factors of geometrically different scattering particles overlap in the low $q R_{g}$ region $\left(q R_{g} \ll 1\right)$. This region is also known as "Guinier regime" and the structure factor in highly diluted systems the structure factor is given by Guinier's law $[1,18]$

Table 2: Particle form factor for a sphere, coil and rod systems.

\begin{tabular}{lrr}
\hline Particle shape & Particle form factor & Reference \\
\hline Sphere & $P_{S}(q)=\left(3 \frac{\sin q R-q R \cos q R}{(q R)^{3}}\right)^{2}$ & {$[10,20]$} \\
Coil & $P_{C}(q)=\frac{2}{u^{4}}\left(e^{-u^{2}}+u^{2}-1\right) \equiv S_{D}(q)^{\mathrm{a}}$ & {$[20,21]$} \\
Rod & $P_{R}(q)=\frac{2}{u^{\prime}}\left(\int_{0}^{u^{\prime}} \frac{\sin x}{x} d x-\frac{2 \sin ^{2} \frac{u^{\prime}}{2}}{u^{\prime}}\right)$ & {$[20,21]$} \\
& & \\
\hline
\end{tabular}

Here $u=q R_{g}$ and $u^{\prime}=q L$ with $L$ length of the rod. a Debye function of a random coil. 


$$
S(q) \approx N\left(1-\frac{q^{2} R_{g}^{2}}{3}+\ldots\right)=N P(q)
$$

In the Guinier regime, one gets information about the size of the diluted polymers, i.e. the radius of gyration $R_{g}$, and the degree of polymerisation of a polymer $N$ can be determined from the absolute intensities. The radius of gyration is the moment of inertia of the scattering object about a specified axis. More precisely, the $z$-average of $R_{g}$ and the weight average of $N$ are obtained by scattering techniques [4].

At large scattering vectors the form factors differ from each other remarkably and the form factor of the spheres shows pronounced minima. From the position of the first minimum the radius of the sphere can be calculated via $q_{\min } R=4.49$. The envelope of the form factor of a sphere is $P(q) \propto q^{-4}$ at large wave vectors, the so called "Porod regime", of a rod it is $P(q) \propto q^{-1}$ and of a coil is $P(q) \propto q^{-2}$ [19].

When neutrons are used as a probe instead of electromagnetic waves, the scattering is induced by the nuclei. Therefore isotopic substitution (e.g. substitution of the hydrogen of the solvent by deuterium) can tune the contrast of the scattering object compared to the solvent. In general the chemical properties are only rather weakly affected by such substitution and the influence on the thermodynamics is considered to be negligible. The strongest contrast is achieved by hydrogen (H) deuterium (D) exchange and therefore HDsubstitution can be systematically varied and different parts of complex structures can be made visible in a controlled way almost without affecting other physical properties. For example, it is possible to understand core-shell structures by visualizing the core and the shell separately [23].

Also the value of the structure function at $q=0$ contains useful information. This value ("forward scattering”) can only be obtained by extrapolation of the scattered intensity from finite $q$-values, as the forward scattered intensity is not measurable directly due to the many orders of magnitude larger transmitted intensity of the primary beam (Fig. 3). Any material, independent of its aggregation state or state of order [i.e. gas, liquid, solid (amorphous, crystalline)], is inhomogeneous in terms of its density. This is obvious from the atomistic nature or all materials. The resulting density fluctuations cause the forward scattering.

From fluctuation theory of statistical thermodynamics it follows that these particle density fluctuations in single component systems are related to the isothermal compressibility $\kappa_{T}[1]$.

$$
S(q \rightarrow 0)=k T \kappa_{T} .
$$

In multicomponent systems, besides the density fluctuations of the individual components also concentration fluctuations are present (and they are of interest!). So an analogous relationship to the isothermal compressibility is the osmotic compressibility $\kappa_{\text {osm }}$, which can be obtained from extrapolating the structure function to $q=0$
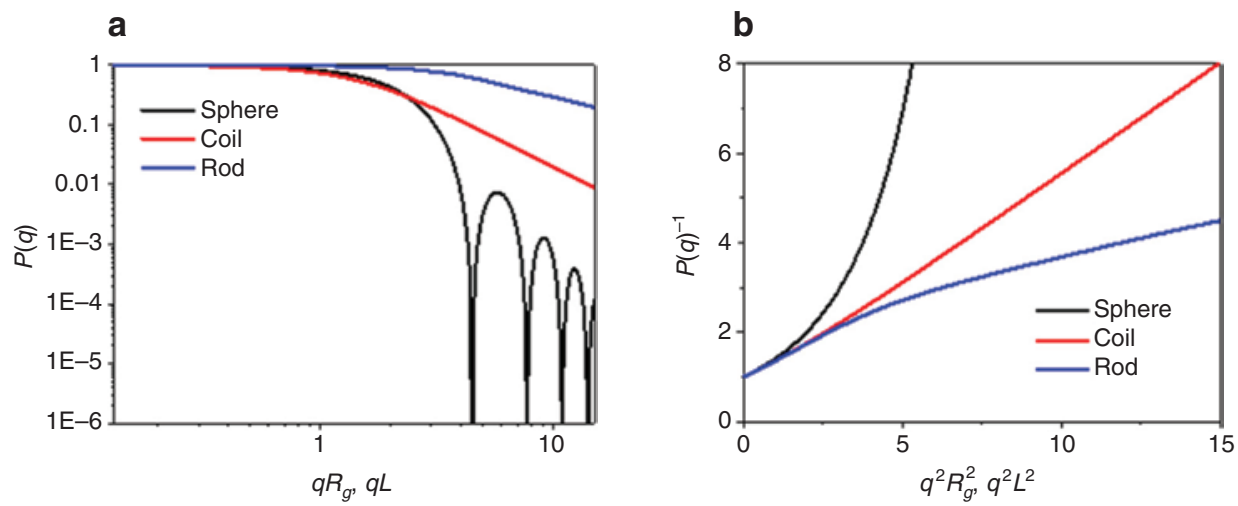

Fig. 2: The scattered intensity of a dilute sample correspond to the particle form factor (eq. 6) which gives information about the shape and size distribution. (a) Particle form factor $P(q)$ for a sphere and a random coil vs. $q R_{g}$ and a rod vs. $q L$. (b) The inverse particle form factor $P(q)^{-1}$ for a sphere, a random coil, and a rod at low scattering vector are plotted vs. the squares of $q R_{g}$ and $q L$, respectively. 


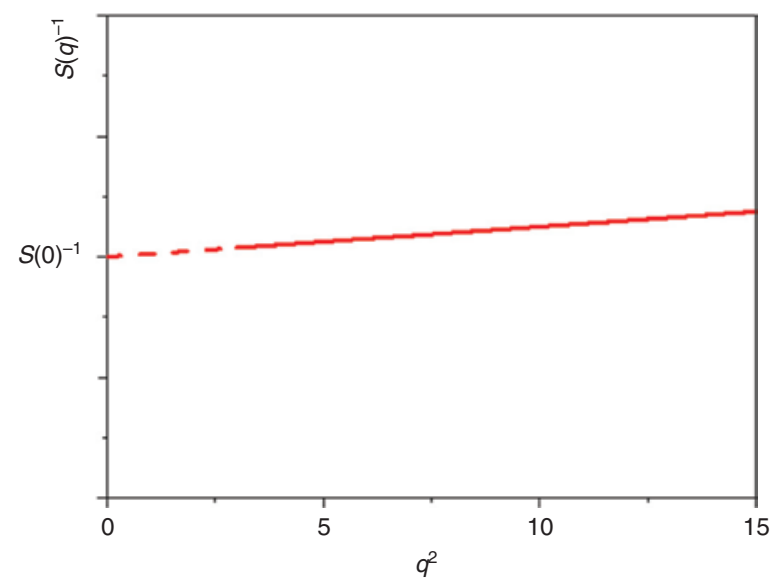

Fig. 3: The inverse structure factor plotted versus the square of the scattering factor $q$. By extrapolating $S(q \rightarrow 0)^{-1}$ the isothermal compressibility $\kappa_{T}$ in case of single component systems (eq. 7) and the osmotic compressibility $\kappa_{\text {osm }}$ in case of polymer solutions (eq. 8) can be determined.

$$
S(q \rightarrow 0)=k T \kappa_{\mathrm{osm}}=k T\left(\frac{\partial c_{m}}{\partial \Pi}\right)_{T} \approx \frac{1}{\left(1+2 A_{2} M c+\cdots\right)}
$$

Here $\Pi=R T\left(c / M_{W}+A_{2} c^{2}+\cdots\right)$ is the osmotic pressure and $\left\langle c_{m}\right\rangle$ denotes the mean value of the monomer density distribution $c_{m}(r)$. The osmotic pressure can be written as a power series in dependence of the molecular weight $M$, the concentration and the second virial coefficient $A_{2}$. The second virial coefficient reflects the interaction between the particles.

To determine the molecular weight $M$ and the second virial coefficient $A_{2}$, the scattered intensity of the sample is measured and normalized by the detector distance $A$, the scattering volume $V$, the incident intensity $I_{0}$. This quantity is the so called Rayleigh ratio $R(q)=1 / V \cdot I(q) / I_{0} A^{2}$. To neglect the fluctuations which arise from the pure solvent, the measured Rayleigh ratio of the pure solvent is subtracted. In order to take into account the geometry of the setup (detector distance, observation angle, ...) the absolute values are determined by calibrating the measured data with a standard sample of known Rayleigh ratio. In case of light scattering often toluene is used due to its large Rayleigh ratio.

Combining the interparticle and intraparticle interactions one gets the so called Zimm-equation [24, 25]:

$$
\frac{K \cdot c}{R(q)}=\frac{1}{M \cdot S(q, c)}
$$

Using eq. 5, 6 with the approximation of $\frac{1}{1-x} \approx 1+x$ for $x \ll 1$ and eq. 7 the Rayleigh ratio gets connected to the radius of gyration and the second virial coefficient.

$$
\frac{K \cdot c}{R(q)}=\left(1+\frac{q^{2} R_{g}^{2}}{3}+\ldots\right)\left(\frac{1}{M_{W}}+2 A_{2} c+\ldots\right) .
$$

A typical Zimm diagram is shown for polyisoprene with a molecular weight of about $185 \mathrm{~kg} / \mathrm{mol}$ in tetrahydrofuran (THF) in Fig. 4.

The ratio $(K \cdot c) / R(q)$ for different concentrations in the range of $1-10 \mathrm{~g} / 1$ was measured. The extrapolation of the ratio $(K \cdot c) / R(q)$ vs. $q=0$ and $c=0$ (red squares) can be linear fitted by

$$
\begin{gathered}
\lim _{q \rightarrow 0} \frac{K c}{R(q)}=\frac{1}{M_{W}}+2 A_{2} c+\ldots . \\
\lim _{c \rightarrow 0} \frac{K c}{R(q)}=\frac{1}{M_{W}}+\frac{1}{M_{W}} \frac{\left\langle R_{g}^{2}\right\rangle}{3} q^{2} .
\end{gathered}
$$




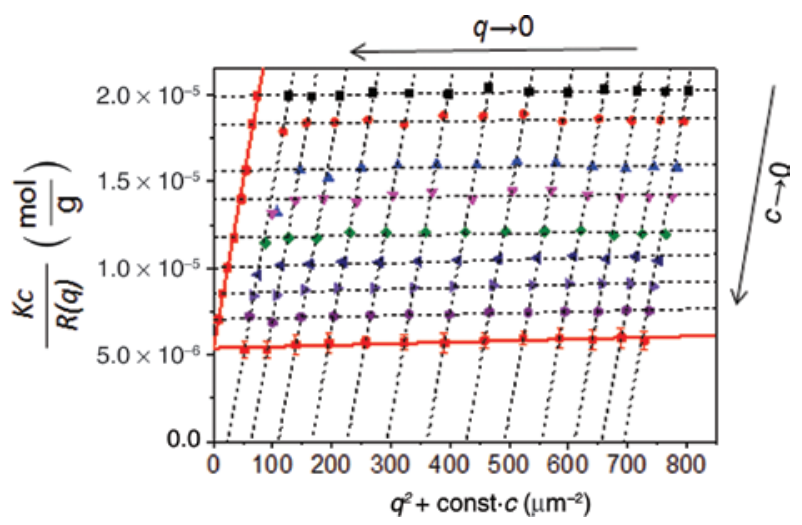

Fig. 4: Light scattering results of polyisoprene in THF. The constant for plotting was set to $10000 \mathrm{l} / \mathrm{mol}^{-} / \mathrm{m}^{-2}$. The same color corresponds to one concentration. The concentration of the samples decreases along the arrow. The red squares are extrapolated values for $c \rightarrow 0$ and $q \rightarrow 0$, respectively. From the $y$-ordinate the inverse weight averaged molar mass can be determined. Here the weight averaged molecular mass was about $185 \mathrm{kDa}$, the radius of gyration was about $10.3 \mathrm{~nm}$ and the second virial coefficient was about $4.310^{-3} \mathrm{~mol} \cdot \mathrm{cm}^{3} \cdot \mathrm{g}^{-2}[26]$.

From the slope of these functions the interaction parameter $A_{2}$ and $R_{g}$ can be calculated. Further extrapolations of equations 11 and 12 vs. $c=0$ and $q=0$, respectively, leads to the intercept $M_{W}^{-1}$.

The second viral coefficient $A_{2}$ adopts different values, depending on the solvent quality. $A_{2}>0$ is indicating a thermodynamically good solvent, in which the dissolved polymer adopts expanded conformations due to the tendency to have many segmental contacts between polymer repeating units and solvent molecules. As a counteracting force, the entropic elasticity force of the polymer favors a more coiled conformation rather than stretched, expanded ones. If $A_{2}=0$, repulsive and attractive interactions between polymer and solvent are balanced. In this so-called theta-condition there is no tendency to expand or contract the chain and the chain adopts the so-called ideal unperturbed conformations of a random coil (ideal coil). Excluded volume effects are compensated by the conformational entropy forces of the chain. Finally, a value $A_{2}<0$ corresponds to a bad solvent and the chains contract and eventually phase separate from the solvent. When studying $R_{g}$ as a function of $N$ for different solvent qualities, different scaling relationships can be found, in agreement with theory. While under theta-conditions the random flight statistics for an ideal coil predicts $R_{g} \propto N^{1 / 2}$, the relationship $R_{g} \propto N^{3 / 5}$ is predicted for a polymer in a good solvent which was derived by de Gennes [1, 27, 28]. In a bad solvent (but polymer is still not precipitated), the relationship $R_{g} \propto N^{1 / 3}$ is found [1]. These relationships could be verified for polymer solutions. An interesting question, however, is the state of a polymer in the melt. Obviously, light scattering and X-ray scattering are not applicable to answer this question due to the absence of contrast. SANS was used to investigate the conformations of a deuterated polymer in a matrix of the similar, nondeuterated polymer. One takes advantage of the fact that the properties of a deuterated chain and a non-deuterated chain are almost the same - with the exception of scattering neutrons. It was found that a polymer in a melt of its own species is under theta-condition. This was already expected by Flory [29] due to the idea that a polymer interacting on a segmental level in a polymer melt cannot distinguish interactions between segments from the same chain and other chains. Therefore there is no driving force for expansion or collapsing of a chain in a matrix of similar chains.

\section{Static scattering of polymer blends and block copolymers[1]}

So far we have considered the static scattering of dilute polymer solutions and in the following we consider the static scattering of polymer blends and block copolymers. We limit the description to binary blends and diblock copolymers. In principle, also more complex systems such as concentrated polymer solutions or blends and block copolymers composed of more than two components can be described, but we only want to highlight some basic principles on as simple as possible examples. Due to the longer wavelength of light this probe is not suitable if different polymers (or polymer blocks) are closely mixed together, even if 
their refractive indices differ significantly from each other. X-rays (if the polymers differ sufficiently in their electron density) and especially neutrons (if deuterated chains can be used as one component) are suitable probes to study the structure of polymer blends and block copolymers. Here we discuss the scattering function only, as its relationship to the measurable scattered intensity is again given by eq. 2 . First we look at the free enthalpy of mixing $\left(\Delta G_{\text {mix }}\right)$ which is often not distinguished from the free energy of mixing $\left(\Delta F_{\text {mix }}\right)$ as the volume changes upon mixing often is negligible in condensed matter (the system behaves incompressible). Flory, Huggins and Staverman published in the same year (1941) a similar lattice-model based description of the free enthalpy of mixing of polymer solutions [30-34]. In literature it is mostly known as the Flory-Huggins theory, which may be due to the fact that Staverman was not allowed to publish in a better recognized international journal due to the occupation of the Netherlands by the German Nazi at that time.

Here we write the Flory-Huggins-Staverman theory in a more generalized form

$$
\Delta G_{\text {mix }}=G_{A B}-\left(G_{A}+G_{B}\right)=R T \tilde{n}_{c}\left(\frac{\phi_{A}}{N_{A}} \ln \left(\phi_{A}\right)+\frac{\phi_{B}}{N_{B}} \ln \left(\phi_{B}\right)+\chi \phi_{A} \phi_{B}\right)
$$

with $\phi_{A}, \phi_{B}$ being the volume fractions of a polymer A and a polymer $\mathrm{B}, \tilde{n}_{c}$ : the molar number of the reference units. $N_{A, B}$, are the degrees of polymerization of the two components A and B assuming that each polymer repeating unit occupies one lattice site, and so does a low molecular solvent if only one of the components is a polymer (i.e. $N_{A}=1$ ). If both components are small molecules, i.e. $N_{A, B}=1$ the volume fractions are equal to molar fractions and the equation describes the free enthalpy of mixing for low molecular liquids. The segmental interaction parameter $\chi=\chi_{S}+\chi_{H}$ includes an entropic contribution given by $\chi_{S}=\frac{\partial}{\partial T}(\chi T)$ and an enthalpic contribution by $\chi_{H}=-T \frac{\partial \chi}{\partial T}$. We just note here that the segmental interaction parameter is also related to the second viral coefficient discussed before $\left(1 / 2-\chi \propto A_{2}\right)$.

The Flory-Huggins-Staverman theory is a mean-field theory and developed by assuming a homogeneous state of mixing, which means that compositional (concentration) fluctuations are not included. In order to include concentration fluctuations, the free enthalpy of a mixture is considered for subvolumes $v_{\text {sub }}$ of the total volume in such a way that these subvolumes are still large enough for statistical thermodynamic considerations, but their composition may deviate from the average. In this way, the total free enthalpy of a mixture with local compositional fluctuations can be described as a sum over the free mixing enthalpies of these subvolumes. Also the "interface" between the different subvolumes contributes to the free enthalpy by an "interfacial energy". As the composition of the mixture is weakly fluctuating, this contribution resulting from a concentration gradient can be expressed by a quadratic term (it cannot be linear, as the sign of the gradient has no influence on the free enthalpy). Higher even orders can be neglected for a weakly fluctuating system [1].

$$
G\left(\left\{\phi_{i}\right\}\right)=\sum_{i} v_{\text {sub }} g\left(\phi_{i}\right)+\sum_{i, j} \beta\left(\phi_{i}-\phi_{j}\right)^{2}
$$

Replacing the sum by an integral leads to

$$
G(\phi(r))=\int\left(g(\phi(r))+\beta^{\prime}(\nabla \phi)^{2}\right) d^{3} r
$$

with $\beta^{\prime}:=\beta v_{\text {sub }}^{-1 / 3}$.

For a constant volume fraction $\phi(r):=\phi$, the fluctuations of the free enthalpy are deviations of the free enthalpy from its minimum: $\delta G:=G-G_{\min }$, and result from fluctuations of the volume fraction $\delta \phi(r):=\phi(r)-\phi$. In this case the fluctuations of the free enthalpy can be expanded up to the second order to:

$$
\begin{aligned}
\delta G & =\int\left(\delta g(\delta \phi(r))+\beta^{\prime}(\nabla \delta \phi)^{2}\right) d^{3} r \\
& =\frac{\partial g}{\partial \phi} \int \delta \phi(r) d^{3} r+\frac{1}{2} \frac{\partial^{2} g}{\partial \phi^{2}} \int(\delta \phi(r))^{2} d^{3} r+\beta^{\prime} \int(\nabla \delta \phi)^{2} d^{3} r
\end{aligned}
$$


By applying the Flory-Huggins-Staverman theory for the fluctuating free enthalpy of mixing and because of conservation of mass $\int \delta \phi(r) d^{3} r=0$, we obtain

$$
\delta G=\frac{R T}{2 v_{c}}\left(\frac{1}{N_{A} \phi}+\frac{1}{N_{B}(1-\phi)}-2 \chi\right) \int(\delta \phi(r))^{2} d^{3} r+\beta^{\prime} \int(\nabla \delta \phi)^{2} d^{3} r
$$

The concentration fluctuations can be expressed in Fourier space and we obtain for the structure function as a function of the scattering vector $q$ :

$$
S(q)=\left(\frac{1}{N_{A} \phi}+\frac{1}{N_{B}(1-\phi)}-2 \chi+\beta^{\prime \prime} q^{2}\right)^{-1}
$$

Comparison with the form factor for low $q$ for very low $\phi$ and $(1-\phi)$, respectively, yields

$$
\frac{1}{S(q)} \approx \frac{1}{N_{A} \phi}\left(1+\frac{\left\langle R_{g, A}^{2}\right\rangle q^{2}}{3}\right) ; \frac{1}{S(q)} \approx \frac{1}{N_{B}(1-\phi)}\left(1+\frac{\left\langle R_{g, B}^{2}\right\rangle q^{2}}{3}\right)
$$

and therefore we write for $\beta^{\prime \prime}$ and the inverse structure function

$$
\begin{gathered}
\beta^{\prime \prime}=\frac{\left\langle R_{g, A}^{2}\right\rangle}{3 N_{A} \phi}+\frac{\left\langle R_{g, B}^{2}\right\rangle}{3 N_{B}(1-\phi)} \\
\frac{1}{S(q)}=\frac{1}{N_{A} \phi}+\frac{1}{N_{B}(1-\phi)}-2 \chi+\left(\frac{\left\langle R_{g, A}^{2}\right\rangle}{3 N_{A} \phi}+\frac{\left\langle R_{g, B}^{2}\right\rangle}{3 N_{B}(1-\phi)}\right) q^{2}
\end{gathered}
$$

This equation was obtained for the low $q$-limit and allows to determine the segmental interaction parameter $\chi$ from the extrapolation to $q=0$, if the degrees of polymerization of the blend partners are known (for example, from light scattering of dilute polymer solutions of the individual blend components). The slope contains contributions from the radii of gyration of both components, which can be determined if at least two blends with different known compositions and known degrees of polymerization are investigated. In Fig. 5a the inverse of the structure factor is plotted vs. $q^{2}$ for different segmental interaction parameters $\chi$. With increasing interaction parameter as well as decreasing temperature the inverse structure factor decreases. The spinodal temperature [this is the temperature where $\partial^{2} G / \partial \phi^{2}=0$ can be extracted by plotting the extrapolated inverse structure factor $S(0)^{-1}$ against the inverse temperature to zero (Fig. 5b)].

However, it can be shown by the so-called Random Phase Approximation that it is possible to extend the description of equation 21 to the whole $q$-range [1]:

$$
\frac{1}{S(q)}=\frac{1}{\phi N_{A} S_{D}\left(R_{g, A}^{2} q^{2}\right)}+\frac{1}{(1-\phi) N_{B} S_{D}\left(R_{g, B}^{2} q^{2}\right)}-2 \chi
$$

For a blend of Gaussian coils the inverse structure factor depends on the inverse form factors (Debye functions) $S_{D}$ of the two blend partners (see Table 2).

Finally we address the structure factor of block copolymers. The most simple case of block copolymers are diblock copolymers where two dissimilar polymer blocks are linked together. Leibler used the Random Phase Approximation to describe the scattering behavior of a diblock copolymer in the disordered state $[36,37]$ :

$$
S(u)=\frac{N}{F(u, \phi)-2 \chi N}
$$



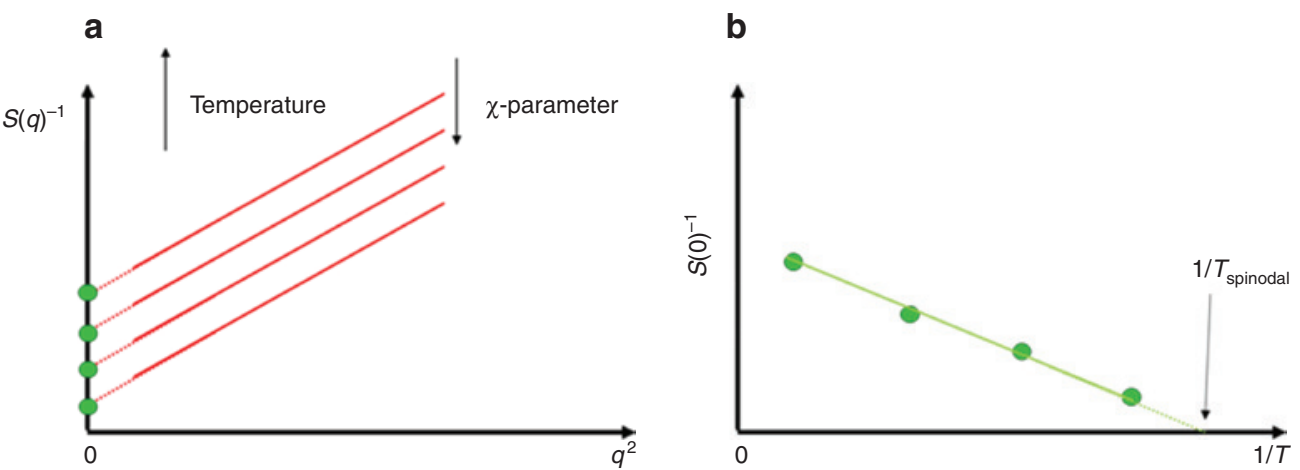

Fig. 5: Determination of the spinodal of a polymer blend with a miscibility gap at lower temperatures from scattering measurements at higher temperatures in the one phase region. (a) Inverse structure factor plotted versus the square of the scattering vector $q$. The $\chi$ parameter increases and the temperature decreases from the upper to the lower curves. (b) The extrapolated inverse structure factor $S(q \rightarrow 0)^{-1}$ plotted against the inverse temperature. From $S(q=0)^{-1}=0$ the spinodal temperature can be obtained.

with

$$
\begin{gathered}
F(\phi, u)=\frac{S_{D}(1, u)}{S_{D}(\phi, u) S_{D}(1-\phi, u)-\frac{1}{4}\left[S_{D}(1, u)-S_{D}(\phi, u)-S_{D}(1-\phi, u)\right]^{2}} \\
S_{D}(\phi, u)=\frac{2}{u^{4}}\left[e^{-\phi u^{2}}-1+\phi u^{2}\right] \\
u=\left(q R_{g}\right)
\end{gathered}
$$

While, as shown before, the structure function of a polymer blend diverges at $q=0$ at the spinodal temperature indicating a macroscopic phase separation, this is impossible for block copolymers due to the fact that their blocks are linked to each other and therefore cannot macroscopically segregate. The resulting microphase separation at the so-called order-disorder transition temperature is indicated by a diverging maximum value at $q>0$ (Fig. 6). The position of the maximum $q^{\star}$ is related to the (sinusoidal) compositional fluctuation wavelength of a diblock copolymer by Bragg's law by $d=2 \pi / q^{\star}$, which also defines the size of the unit cell in the ordered state, in which the microdomains are assembled in crystal-like lattices. Higher order maxima indicate the symmetry and structure of the ordered phase similar to X-ray diffraction patterns of low molecular weight crystals. The larger is the repulsion between dissimilar segments of the block copolymer, the stronger the different blocks segregate from each other leading to more stretched chains. This can be expressed by the product of the degree of polymerization $N$ and the segmental interaction parameter $\chi$. Already in the disordered state $(\chi N<10)$ where entropy dominates repulsive interactions between the different blocks, $\chi N$ influences the relationship between the degree of polymerization of the block copolymer and the compositional fluctuation wavelength. According to the theory of Leibler, which describes the so-called weak segregation between blocks the compositional fluctuation wavelength scales with the square root of the degree of polymerization:

$$
d \propto N^{1 / 2}
$$

In strongly segregated block copolymers the compositional fluctuation wavelength approaches a boxcar function with sharp interfaces and the scaling relationship between degree of polymerization and the periodic length scale of the microphase morphology is

$$
d \propto \chi^{1 / 6} N^{2 / 3} .
$$




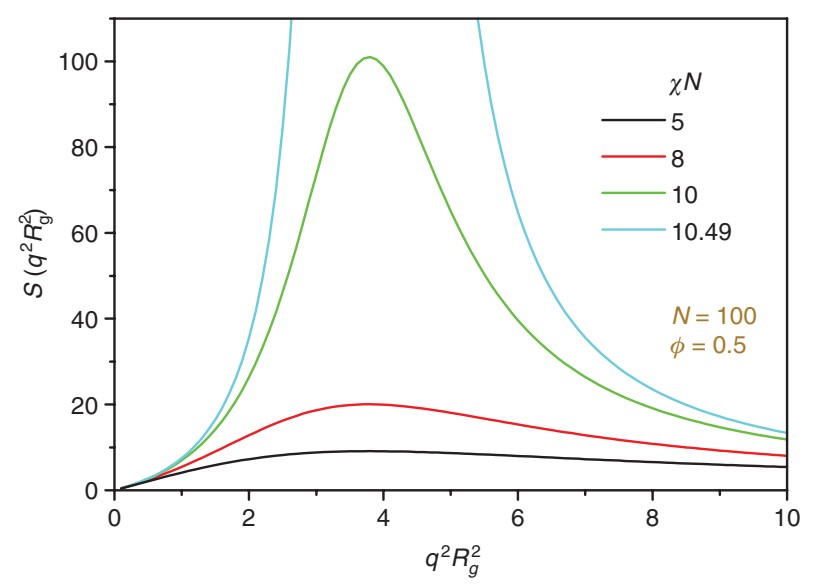

Fig. 6: Structure factor for a diblock copolymer with symmetric composition $(\phi=0.5)$. The maximum occurs at the position of 3.873 according to the theory of Leibler and allows the determination of the radius of gyration of the whole block copolymer chain. The divergence of the maximum to infinity when the system transforms from the disordered to the ordered phase is a result of the approximations of this theory but cannot happen in reality. So-called fluctuation corrections can account for this [35].

The thickness of the interface between strongly segregating blocks $w$ also depends on the interaction parameter:

$$
w \propto \chi^{-1 / 2}
$$

Matsen and Bates gave a more complete description of the theory of microphase separation in block copolymers including both weak and strong segregation using self-consistent field theory (SCFT), which is beyond the scope of this paper [38].

\section{Dynamic scattering of dilute polymer solutions}

Dynamic light scattering is a standard tool to determine the hydrodynamic size of the particles via the Brownian motion [6,39]. Due to the Brownian motion the density fluctuates within the sample volume (Fig. 7a). This changes the interference pattern and the measured intensity fluctuates (Fig. 7b). In a typical experiment the intensity is measured at a defined angle (usually at $90^{\circ}$ ). The diffusion coefficient is related to the field autocorrelation function $g_{1}(q, \Delta t)$. However, the field autocorrelation function is normally not directly measured. In a typical light scattering setup the intensity is measured and a correlator determines directly the intensity autocorrelation function $g_{2}(q, \Delta t)$ (Fig. 7c). The intensity autocorrelation function is the normalized temporal average of the product of the intensity at at time $t$ and $t+\Delta t$

$$
g_{2}(q, \Delta t)=\frac{\langle I(q, t) I(q, t+\Delta t)\rangle_{t}}{\left\langle I(q, \Delta t)^{2}\right\rangle_{t}}
$$

The brackets $\langle\ldots\rangle_{t}$ denote the time averaged value.

If the intensity is still correlated the normalized intensity autocorrelation function is equal to 2 (Fig. 7c). When the intensity starts to decorrelate the normalized intensity autocorrelation function drops exponentially to 1 . The time it takes to decorrelate is the so called relaxation time.

To determine the relaxation time the field autocorrelation function is needed.

The field autocorrelation function $g^{1}(q, \Delta t)$ can be determined via the Siegert relation [39]

$$
g_{1}(q, \Delta t)=\sqrt{g_{2}(q, \Delta t)-1}=\frac{S(q, \Delta t)}{S(q, 0)}=f(q, \Delta t)
$$


a

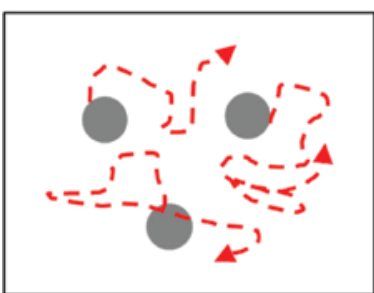

b

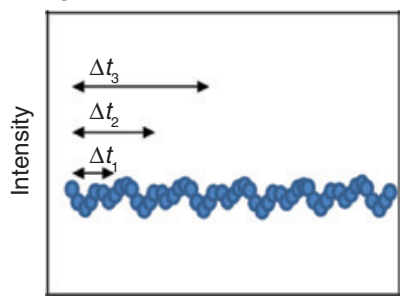

Time $t$ (a.u.)

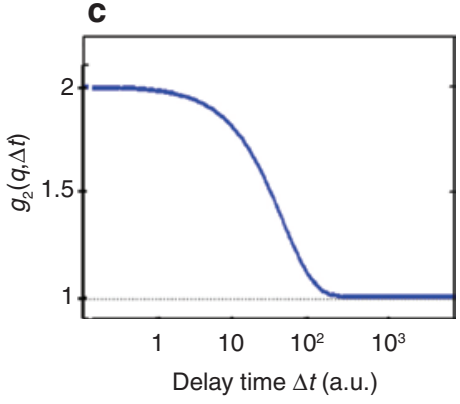

Fig. 7: Due to the Brownian motion of the particles the diffusion coefficient can be determined via the intensity autocorrelation function $g_{2}(q, \Delta t)$. (a) Brownian motion of the particles within the sample volume. (b) Measured intensity plotted vs. the time. (c) Intensity autocorrelation function $g_{2}(q, \Delta t)$ versus the delay time $\Delta t$. At short time scale the intensity is correlated and equal to two. When the intensity starts to decorrolate the intensity drops exponentially to one.

The field autocorrelation function $g_{1}(q, \Delta t)$ is also called dynamic structure factor $f(q, \Delta t)$ which is the ratio of the structure factor at a time $\Delta t$ to $\Delta t=0$. The dynamic structure factor can be described in case of dilute systems with a simple exponential decay function with the relaxation time $\tau$.

$$
f(q, \Delta t)=\exp \left(-\frac{\Delta t}{\tau}\right)
$$

In case of Brownian motion the diffusion coefficient is connected to the relaxation rate $\Gamma=\frac{1}{\tau}$ via

$$
D=\Gamma q^{2} .
$$

Using the Stokes-Einstein relation, the hydrodynamic radius $R_{\text {hyd }}$ can be obtained from the diffusion coefficient:

$$
D=\frac{k_{B} T}{6 \pi \eta R_{\text {hyd }}}
$$

Here $k_{B}$ is the Boltzmann constant and $\eta$ the viscosity of the solvent. The hydrodynamic radius is the geometric radius of an equivalent solid sphere which would show the same dynamic scattering behavior. With other words, this information alone will not give a detailed information of the shape of the scattering polymer. More information about the anisotropy of the shape of the scattering structures can be obtained by using different linear polarizations both for incident and detected light, what is beyond the scope of this paper $[39,40]$. There are many applications of dynamic light scattering. For example, from temperature dependent dynamic light scattering it is possible to observe aggregation phenomena in dilute polymer solutions when they approach the precipitation temperature [41]. By combining DLS with static light scattering one can determine the ratio of $\left(R_{g} / R_{\text {hyd }}\right)$. This ratio gives information about the shape of the particles, which was done i.e. for thermoresponsive hydrogels to understand the swelling behavior [42].

\section{Further applications and outlook}

In this paper we presented some basic relationships between the structure factor and thermodynamic quantities of polymers in bulk state and solution, as well as dynamic properties in polymer solutions. In general scattering experiments include larger sample volumes as compared to microscopic measurements and therefore scattering methods allow to get a more averaged information about the homogeneity of the sample compared to microscopy, unless many pictures are taken by microscopy. However, we emphasize that for structural investigations microscopic methods and scattering methods have their benefits, while thermodynamic properties are accessible only with the latter in a straight forward manner. 
While we presented studies on homogeneous (single phase) systems before, it should be stated that also the structure of heterogeneous polymer blends can also be studied by SAXS, SANS, and light scattering. For example, the quasi periodic length scale of spinodally decomposing blends and their time dependence can be investigated [43] as well as the thickness of the interfacial layers between the coexisting phases [44].

Also the combination of SAXS and SANS measurements can be very useful. For example, a macroscopically aligned lamellar microphase separated block copolymer was characterized by SAXS in terms of orientation of the structure. SANS on the same sample revealed the anisotropic stretching of a deuterated polymer block perpendicular to the microphase boundary [45]. Such a result can hardly be obtained by microscopic techniques. Very complex morphologies like quasi crystals in assemblies of block copolymer micelles could be only proven by a combined investigation using SAXS and SANS [46].

An interesting development is the combination of scattering techniques with other techniques in situ, i.e. the combination of SAXS or SANS with rheological measurements [47-49] or with electric fields [50]. In this case the structural origin of the mechanical properties can be investigated. Also the structure formation during solvent evaporation from a block copolymer solution can be investigated [51]. With the development of the synchrotrons of the third generation and the new light sources [52, 53], the properties of the synchrotrons improved like the coherence, the spatial as well as temporal resolution. Using the coherence properties of the third generation synchrotrons or X-ray free electron lasers makes it possible to expand the dynamical investigation also to X-rays [54]. This offers the possibility to investigate the dynamics at smaller time and length scales for even optically opaque samples. These new possibilities were demonstrated for a polymer melt, in which the entanglements were measured with the help of gold tracer particles [55].

With this paper we showed that scattering techniques are already present for a long time and they continuously improve and new possibilities evolve. Therefore we hope to encourage scientists to consider also scattering methods when they are interested to know more about the thermodynamic properties, the structure, or the dynamics of polymer systems.

\section{References}

[1] G. Strobl. The Physics of Plymers Concepts for Unterstanding Their Structures and Behavior, Springer, Heidelberg (2010).

[2] C. C. Han, A. Z. Akcasu. Scattering and Dynamics of Polymers: Seeking Order in Disordered Systems, John Wiley \& Sons, (Asia) Pvt Ltd., Singapore (2011).

[3] M. Rubinstein, R. H. Colby. Polymer Physics, Oxford University Press, Oxford, UK, (2003).

[4] P. C. Hiemenz, T. P. Lodge. Polymer Chemistry, CRC Press Taylor \& Francis Group, Boca Raton (2007).

[5] O. Glatter, O. Kratky. Small Angle X-ray Scattering, Academic Press, London (1982).

[6] W. Schärtl. Light Scattering from Polymer Solutions and Nanoparticle Dispersions, Springer, Berlin, Heidelberg, New York (2007).

[7] G. L. Squires. Introduction to the Theory of Thermal Neutron Scattering, Dover Publications, Mineola (1997).

[8] T. Zemb, P. Lindner. Neutrons, X-rays and Light: Scattering Methods Applied to Soft Condensed Matter, North-Holland (2002).

[9] J. S. Pedersen. Neutrons, X-Rays and Light: Modelling of Small-Angle Scattering Data from Colloids and Polymer Systems, pp. 391-420, Elsevier Science, Amsterdam, The Netherlands (2002).

[10] L. Rayleigh. Proc. Roy. Soc London Ser. A 84, 25 (1910).

[11] G. Mie. Ann. Phys. (Leipzig) 25, 377 (1908).

[12] E. Rutherford. Philos. Mag. Series 6, 21, 669 (1911).

[13] N. B. Colthup, L. H. Daly, S. E. Wiberley. Introduction to Infrared and Raman Spectroscopy, Academic Press, New York (1990).

[14] K. Kneipp Y. Wang, H. Kneipp, L. T. Perelman, I. Itzkan, Irving, R. R. Dasari, and M. S. Feld. Phys. Rev. Lett. 78, 1667 (1997).

[15] P. Eisenberger, P. M. Platzman. Phys. Rev. A 2, 415 (1970).

[16] N. Stribeck, X-Ray Scattering of Soft Matter, Springer, Berlin, Heidelberg (2007).

[17] M. Kotlarchyk, S. H. Chen. J. Chem. Phys. 79, 2461 (1983).

[18] A. Guinier, G. Fournet. Small Angle Scattering of X-rays, John Wiley \& Sons. Inc., New York (1955).

[19] B. Hammouda. A Tutorial on Small-Angle Neutro Scattering from Polymers, National Institute of Standards and Technology, Gaithersburg (1995). L. Rayleigh. Proc. Roy. Soc London, Ser. A 84, 25 (1910).

[20] J. S. Pedersen. Adv. Colloid Interface. Sci. 70, 171 (1997). 
[21] T. Neugebauer. Ann. Phys. 42, 509 (1943).

[22] P. Debye. J. Phys. Colloid Chem. 51 (1947).

[23] D. Pötschke, M. Ballauff, P. Lindner, M. Fischer, F. Vögtle. Macromolecules 32, 4079 (1999).

[24] B. H. Zimm. J. Chem. Phys. 16, 1093 (1948).

[25] B. H. Zimm. J. Chem. Phys. 16, 1099 (1948).

[26] E. Rahmstorf. Synthesis and Hydrolysis of Polyisopreneblock-poly(Tert-butyl Methacrylate), and its Hydrolysis to Poly(Methacrylic Acid), master thesis, University of Hamburg, (2014).

[27] P. J. Flory. J. Chem. Phys. 17, 303 (1949).

[28] W. Kuhn. Koiloid-Zeitschrift 68, 2 (1934).

[29] P. J. Flory. Principles of Polymer Chemistry, George Banta Publishing Company, Menasha/Wisconsin (1953).

[30] P. J. Flory. J. Chem. Phys. 9, 660 (1941).

[31] P. J. Flory. J. Chem. Phys. 10, 51 (1942).

[32] J. Staverman, J. H. V. Santen. Recueil des Travaux Chimiques des Pays-Bas 60, 76 (1941).

[33] M. L. Huggins. J. Chem. Phys. 9, 440 (1941).

[34] P. J. Flory. Statistical Mechanics of Chain Molecules, Interscience Publishers, University of Michigan (1969).

[35] G. H. Fredrickson, E. Helfand. J. Chem. Phys. 87, 697 (1987).

[36] L. Leibler. Macromolecules 13, 1602 (1980).

[37] Y. Erukhimovich, A. R. Khokhlov, T. A. Vilgis, A. Ramzit, F. Boue. Comput. Theor. Polym. Sci. 8, 133 (1998).

[38] M. W. Matsen, F. S. Bates. J. Chem. Phys. 106, 2436 (1997).

[39] B. J. Berne, R. Pecora. Dynamic Light Scattering: With Applications to Chemistry, Biology, and Physics, Dover Publications, New York (2003).

[40] D. Lehner, H. Lindner, O. Glatter. Langmuir 16, 1689 (2000).

[41] S. Eggers, B. Fischer, V. Abetz. Macromol. Chem. Phys. 217, 735 (2016).

[42] X. Wang, C. Wu. Macromolecules 32, 4299 (1999).

[43] T. Hack, V. Abetz, M. Stamm, D. W. Schubert, K. Mortensen, W. Siol. Colloid Polym. Sci. 274, 350 (1996).

[44] T. Hashimoto, M. Takenaka, H. Jinnai. J. Appl. Cryst. 24, 457 (1991).

[45] H. Hasegawa, T. Hashimoto, H. Kawait, T. P. Lodge, E. J. Amis, C. J. Glinka, C. C. Han. Macromolecules 18, 67 (1985).

[46] S. Fischer, A. Exner, K. Zielske, J. Perlich, S. Deloudi, W, Steurer, P, Lindner, S. Förster. PNAS. 108, 1810 (2011).

[47] T. Meins, K. Hyun, N. Dingenouts, M. Fotouhi Ardakani, B. Struth, M. Wilhelm. Macromolecules 45, 455 (2011).

[48] B. Struth, K. Hyun, E. Kats, T. Meins, M. Walther, M. Wilhelm, G. Grübel. Langmuir 27, 2880 (2011).

[49] J. Stellbrink, B. Lonetti, G. Rother, L. Willner, D. Richter. J. Phys. Cond Matter 20, 404206 (2008).

[50] K. Schmidt, A. Böker, H. Zettl, F. Schubert, H. Hänsel, F. Fischer, T. M. Weiss, V. Abetz, A. V. Zvelindovsky, G. J. A. Sevink, G. Krausch. Langmuir 21, 11974 (2005).

[51] C. Stegelmeier, A. Exner, S. Hauschild, V. Filiz, J. Perlich, S. V. Roth, V. Abetz, S. Förster. Macromolecules 48, 1524 (2015),

[52] B. W. J. McNeil, N. R. Thompson. Nat. Photonics 4, 814 (2010).

[53] M. E. Couprie. J. Electron Spec. Rel. Phenomena 196, 3 (2014).

[54] M. Sutton, S. G. J. Mochrie, Greytak, S. E. Nagler, L. E. Berman, G. A. Held, G. B. Stephenson. Nat. Lett. 352, 608 (1991).

[55] J. Carnis, W. Cha, J. Wingert, J. Kang, Z. Jiang, S. Song, M. Sikorski, A. Robert, C. Gutt, S. W. Chen, Y. Dai, Y. Ma, H. Guo, L. B. Lurio, O. Shpyrko, S. Narayanan, M. Cui, I. Kosif, T. Emrick, T. P. Russell, H. C. Lee, C.-J. Yu, G. Grübel, S. K. Sinha, H. Kim. Sci. Rep. 4, 6017 (2014). 